\title{
A Review on Organizational Modules of Autonomous Vehicles
}

\author{
Kunal Mehta \\ Student (Computer \\ Engineering) \\ Mukesh Patel School of \\ Technology Management \\ and Engineering \\ Mumbai, Maharashtra \\ 400056
}

\author{
Vineet Makharia \\ Student (Computer \\ Engineering) \\ Mukesh Patel School of \\ Technology Management \\ and Engineering \\ Mumbai, Maharashtra \\ 400056
}

\author{
Rumil Dand \\ Student (Computer \\ Engineering) \\ Mukesh Patel School of \\ Technology Management \\ and Engineering \\ Mumbai, Maharashtra \\ 400056
}

\author{
Prachi Natu \\ Assistant Professor \\ Mukesh Patel School of \\ Technology Management \\ and Engineering \\ Mumbai, Maharashtra \\ 400056
}

\begin{abstract}
Autonomous vehicle technology has seen immense breakthroughs in recent years. Currently existing models, however, are inoperable in moderate to highly adverse environments, clearly indicating a need for refinement in all three key aspects of a self-driving vehicle, which are navigation, system interconnection and security systems. A self-driving vehicle must be able to traverse difficult terrain in adverse weather conditions and reach its destination accurately before its navigation systems can be deemed fit for public usage. Self-driving vehicles must be interconnected and must work efficiently in cooperation to minimize traffic and time delay in travel while also having adequate backup systems to cope with interconnection and network failures. In this paper are reviewed multiple different approaches to the solution of each of these aspects of a self-driving car. Based on the inferences of this review, the areas where improvement could result in more breakthroughs which could make autonomous vehicle technology get implemented in the near future are highlighted.
\end{abstract}

\section{Keywords}

Cybersecurity, intrusion detection, navigation, self-driving car, system-of-systems, Voronoi cells.

\section{INTRODUCTION}

An autonomous car is a vehicle that is adept at sensing its surroundings and navigating without human involvement [1]. They have immense potential to allow for the more productive use of time spent in vehicles and to reduce crashes, congestion, energy consumption, and pollution. Teslas autopilot system on its Model S and Google Chauffeur and similar breakthroughs in the field supplemented by the DARPA (Defense Advanced Research Projects Agency) Grand and Urban Challenges, to name a few, have made autonomous driving technology a subject of considerable public interest over the last decade. Another compelling factor

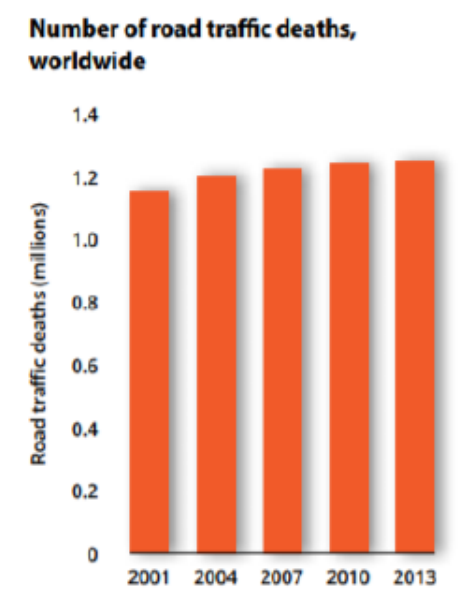

Figure 1: Global status report on road safety by World Health Organization 2015 [2]

is the alarming statistic that lives lost in road accidents worldwide have plateaued at 1.25 million annually as of 2015 [2]. This means that every 25 seconds a human life was lost in vehicular traffic related tragedies. Googles driverless vehicles, on the other hand, have covered over 7,00,000 miles having been involved in 2 incidents only, both of which in fact was the result of human error on the part of the other vehicles drivers. Bearing all of these factors in mind, self-driving vehicles are the next progressive step in the transportation sector.

\section{COMPONENTS}

There are four broad components of a self-driving vehicle. They are navigation, the network connecting self-driving cars, roadside curb detection and security systems. Navigation is chiefly concerned in getting from the source location to the destination. Path planning, collision detection, and environmental scanning systems all work together to achieve the shortest and safest path from the source to the destination without any collisions or detours. Networks refer to both the interconnected web between all self-driving cars that enable more efficient and cooperative navigation, and the networking connections vital to input via GPS and other sources as well. Security systems are the most crucial of each of these components since, without robust and dynamic security systems in place, self-driving vehicles will be vulnerable to 
remote access and control by malicious sources which could be a potential disaster.

\subsection{Navigation}

Self-driving vehicles remain a distant dream until vehicles can handle all different terrains and navigate safely and reliably in adverse weather conditions. Cars are driven in all geographic locations with varied weather conditions. In order to enable autonomous cars to handle adverse driving conditions like wet roads, snow, etc, the navigation algorithm must be able to detect roads which would allow a tolerable margin of error in navigation with minimum risk [3]. Sensor fusion algorithms[4][3][5] combat these issues and were coincidentally validated in the 2014 Hyundai Motor Companys Autonomous Car Competition [3][5]. The setups involve Velodyne HDL-32E LiDARs, Ethernet color cameras and are implemented on vehicles, all of which are controlled by multiple computers and industrial PCs connected by Gigabit Ethernet for fast communication of large image and laser scan data [3][6][5]. The information from all these sources enables the vehicle to detect the lane more robustly even when the car has to navigate in rain.

Instead of heavy input based planning as in [3], the algorithms proposed in [7], in the last few years, model-based path planning has emerged as an effective solution to realtime path planning because it takes into account the kinematics of the vehicle. The algorithm involves Hermite interpolation [7] based connecting trajectory generation methods. The algorithm consists of two parts: online trajectory generation and selection. The trajectory generation method is improved by considering a kinematic model of a car-like vehicle [4]. The terminal state setup is improved according to current vehicle state and road information. In addition, performance index in the referenced paper took connecting trajectory between start and goal as a straight line, which is unreasonable when the road is a curve, so a Hermite interpolation based connecting trajectory generation method is proposed considering initial and goal heading. The vehicle kinematic model is given, as shown in Fig.2, by the three basic vector equations [7]:

$$
\begin{aligned}
& \mathrm{x}=\mathrm{v} \cdot \cos \theta \\
& \mathrm{y}=\mathrm{v} \cdot \sin \theta \\
& \theta=\frac{\mathrm{v} \cdot \tan \beta}{\mathrm{l}}
\end{aligned}
$$

Yet another completely different approach is the integration of Voronoi cells [8]. The algorithm is composed of three parts: the novel path representation, the collision detection and the path modification using a Voronoi cell. The novel path representation provides convenience for checking the collision and modifying the path and a continuous control input for steering wheel rather than waypoint navigation. The algorithm was applied to Eurecar KAIST, thereby validating the systems in real time. Voronoi cell concepts are used and then decomposed promptly into systems of cubic equations to guide the car. The roadmap based approach is divided into the

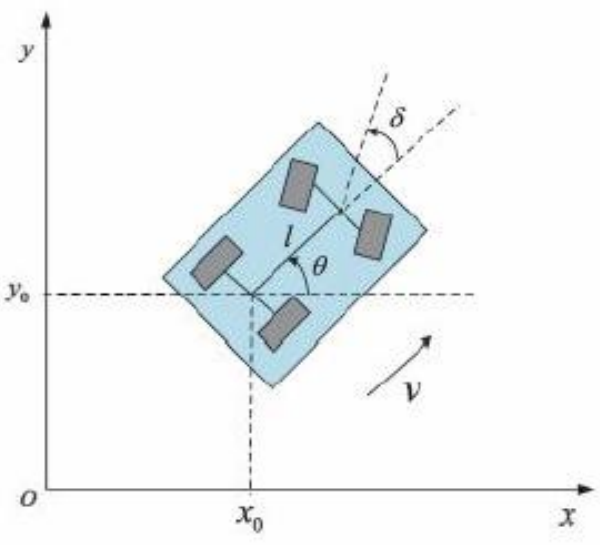

Figure 2: The vehicle model [7]

deterministic roadmap and probabilistic roadmap. A typical example of the probabilistic roadmap is using RRT. In this approach, an algorithm that considers the non-holonomic motion of vehicles is widely used. However, in order to process the RRT algorithm in real time, high computing is required and its result is highly dependent on heuristics. The vehicle is equipped with two DGPSs from Novatel Company, IMU (Inertial Measurement Unit) and industrial PC104 for navigation, eight laser scanners for collision detection and five cameras for image processing. Three PCs equipped with Intel i7 CPUs were used for laser data and image signal processing, path management and generation, and control input computation [9]. To manipulate the vehicle, an actuator was mounted to throttle and brakes while the handling used MDPS controlled by CompactRio [9].

A huge majority of all the current headway being made in the navigation aspect of self-driving vehicles involves either one or a combination of these approaches. However, there are a lot of issues yet to be addressed with precision. The algorithm proposed in [3] vulnerable in sharp turn conditions because navigation there requires correction in the tangential direction of the path because of the RANSAC based plane fitting that causes lanes to be detected on the path grid of the initial normal direction of the path. Turning corrections are possible but these compromise on lane detection because of the obscured view of the lane and the road beyond the turn. Model-based path planning algorithms [1][7] are ideal scenario based systems, which have no backup in case of loss of data connections. While they reduce the need for hardware inputs, they're mathematically complex. Path management using cubic formulae [9] is more efficient and path modification is easier than waypoints, but path planning using the Voronoi diagram is not suitable for real-time application due to taking a calculation time in an algorithm that searches the Voronoi diagram and the optimal path. Thus it is not appropriate to apply this to point cloud directly which is obtained from real laser scanners. The study assumes no slip between wheels and the surface on which the car is driving which is not practical for real-time usage [9][7][3][10][1]. Also, all of the discussed models assume left and right front wheels to be equivalent as in an ideal case to control the lateral direction of a vehicle. In reality, this is rarely the case, a little bit of subconscious fine tuning is done by the human drivers. 


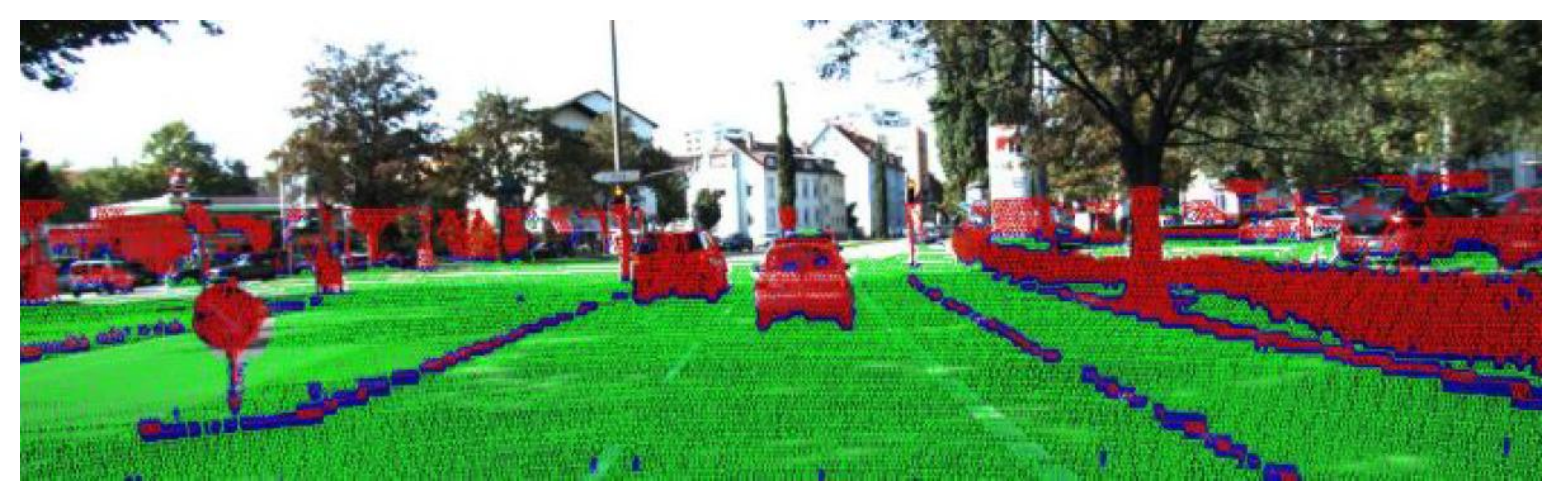

Figure 3: Red represents edges between two points classified as obstacle, green represents edges between nonobstacle points, and blue represents edges between non-obstacle points and obstacle points [12].

\subsection{Network of Self-Driving Cars}

One of the main advantages of having autonomous vehicles is the interconnection [11] of all the vehicles commuting and all of them communicating and interacting in a way to optimize collective transportation goals by making it quick, effective and efficient for all the vehicles in the collective system. The paper [11] sheds some light on the aspect of topologies in a network of autonomous vehicles. It treats the car as a fully functional system and all the functionalities and independent modules as part of a network that helps in smooth functioning of the car. This network can be observed on a granular view of the system. A larger picture that has all the cars as individual nodes communicating on the network of autonomous vehicles to collectively maintain motion by regulating speeds and breaking over a huge mass of moving vehicles ensuring minimal or no traffic and swift overall commute is proposed. An outline of the local and the networking capabilities of the system is provided and takes a comprehensive step by step analysis of each of the necessary components. Taking the car as a system, we have 14 major components [11]. They are: Actuation system, Path Planning, Position Determination, Emergency Response, Analysis, Maintenance and Diagnostics, Command, Communications, Object and Environment Recognition, Manual Override, Local Map, Peer Interaction, Non-Cooperative vehicle interaction and Sensing System.

Path Planning, Position Determination, Local Map, Sensing System, Object and Environment Recognition act at the root level, determining the navigation to the destination as well as local movement on the road based on external actors and factors. They dictate the behaviour of the autonomous vehicle as an individual machine. Peer Interaction and Communications ensure that the cars form a network and work together. In an ideal setting, exceptions are unlikely to occur but due to a certain error rate introduced by human drivers as well as unexpected circumstances contingency systems are also in place as Maintenance and Diagnostics and Sensing Systems. Their job is to predict and catch any on road exception that could lead to potential harm and alert the system about it. Non- Cooperative Vehicle Interaction, Emergency Response and Manual Override are certain specialized modules that receive the alert and depending upon the situation, corrective measures are implemented.

Certain components are aimed at multi vehicle coordination system. They are: Other cars plan and position determination, Disaster Response and Non cooperative vehicle prediction system. Since the position and plan of the journey is shared with the vehicles surrounding the main vehicle, it is the path of a multivehicle system. Disaster response for a system is different as it analyses the danger with respect to the entire group of cars that are present and how to minimize the damage to the system instead of just protecting an individual. There are certain other factors that are taken into account as well which are independent of the vehicle, yet are necessary for the proper functioning of the system. These factors take care of things like the Environment surrounding the path or the area of travelling, the Road Side Units (RSUs) [11] that help in regulating the movement of cars and act like checkpoints. Map integration is a pivotal aspect in maintaining order at scale.

The given paper [11] comprehensively covers all the various concepts that help the autonomous vehicle function at the node level as well as the network level. The paper is extremely exhaustive in formulating all the aspects of a fully autonomous system but it doesn't leave room for uncertainty. It proposes a system that would essentially work after total transition has occurred to autonomous transportation. The human element and the uncertain nature of transport, which will be the working environment for the system, makes it difficult for the model to sustain in unpredictable environment with a lot of human factors at work which can lead to destabilization. There needs to be more focus on assisted driving with a human component as expecting a total transition at such a rate is near impossible and so is implementing an ideal world system in the real world.

\subsection{Roadside Curb Detection}

Obstacle Detection [8][12] is one of the most fundamental need of an autonomous vehicle. Object detection is the basic decision making process that helps the car decide the next move at a much smaller scale. While Path Planning and Road Mapping are entirely based on existing roads, object detection is entirely dynamic as the car is supposed to visualize its surroundings and realize the number of options it has in travelling to its desired location while ensuring its own as well safety of others in the vicinity. Another method of road terrain detection is the usage of 3D Light detection and Ranging technique (LiDAR) technology for the plotting of obstacles and Sensor Fusion for obstacle detection [12]. LiDAR technique is used to plot the $3 \mathrm{D}$ environment to a $2 \mathrm{D}[8][12]$ screen using sensor fusion [12][5] to determine the type and importance of each pixel with respect to the decisions to be made. 
It follows a 5 step process that begins with Sensor Fusion which is plotting of the points. Next follows graph generation which joins all the pixels which are connected in the 3D space to establish the relationship between them. Then each pixel mass is classified as obstacle or not (obstacle classification). Obstacle points, Non-obstacle points and their interfaces are all marked in different colour as shown in figure 3. This information is then later used in the 4th step which is free space detection which helps in determining the available free spaces on the given road. The last step is the road estimation using the histograms generated in the 4th step which provides the exact idea of how much road the car can cover or has the option to. The given method was experimentally and theoretically tested. Despite performing exceptionally in the theoretical domain, there were still certain issues in recognizing the delimiting values between the road and the curb hence having a lot of room for improvement. A model of sensor fusion detects obstacles dynamically in an effective manner [8][12]. While there can be various methods of plotting the 3 dimensional data to 2 dimensions for the car to interpret, the algorithm that is used to detect the obstacles and free spaces needs to be improved upon so that the decision making for the path to be taken is safer and quicker. Quicker decisions would leave room to apply corrective measures for any unforeseeable events that might occur to maim the car or the system in general.

\subsection{Cybersecurity for Self-Driving Cars}

The advantages of an interconnected system are explained above. However, an interconnected system has a risk of being hacked into and the intruders gain control of the self-driving vehicles in the network. Not only hacking, but network failures can be another vulnerability in an interconnected system. Hence, security and maintenance of this system are of paramount importance. The system of interconnected cars is most vulnerable to a black hole or packet drop attack [14]. It is a type of denial-of-service (DOS) attack. Black-hole attacks are a very effective and quick way to gain control, destroy or compromise any given network. These attacks take hold of one node and either start dropping packets or change their vector table in such a way that they have the least traffic [14]. This way they can grab hold of a network just by grabbing a single node out of it and finding the infected or malicious node is difficult because of the ad hoc and ever-changing nature of the network of self-driving cars. This can cause a major problem in an interconnected system. Thus, it is desirable to develop a system to secure the vehicles, the protocols, and prospective issues before they cause an accident or an injury. The paper [8] tries to propose a model for the network security of the interconnected system. It comprises of 4 layers-

1. Vehicle Level Intrusion Detection System (IDS)- It is one of the most simple and basic levels in the model. The system performs the analysis using the data collected by the car itself. The foundation of this level is mutually verifying information. This essentially means that a car checks the information that is being transmitted by the other node using its own sensors (like LIDAR). If the transmitted information does that match the sensed data, then the locally sensed data will be given priority over the received information.
2. Vehicle Area Network (VANET) Intrusion Detection- A reputation rating is assigned to all cars in the VANET. If the reputation rating is lesser than a threshold, that node is deemed suspicious and the vehicles stop receiving data from that node, as shown in Fig.4. In the emergency braking scenario (a), a node is transmitting information of a crash ahead of it to the other nodes. However, the false scenario (b) represents the actual scenario. In this case, the reputation rating of the node will decrease.

3. Wide Area Vehicle Coordination and Traffic Management Intrusion Detection- Traffic Management Intrusion detection is divided into 2 parts- Roadside Units(RSUs) and the VANETs. Each RSU correlates the information it receives from nodes within the VANETs. It sorts out malicious node messages and passes relevant information to other VANETs over a wider area. Involving the RSU is beneficial as it is able to perform a more thorough evaluation of the node, based on a larger dataset than a particular VANET could have access to.

4. Homeland Security or any other government agency- The IDS to protect against a high level of attack utilizes a combination of a centralized, hierarchical, and distributed mechanisms. It includes a central data handler [8] that communicates with all RSUs (collecting information, in turn, from VANETs and individual nodes). It secures the interconnected network of RSUs and looks for critical issues with the system. This is a final failsafe that ensures that no widespread damage can occur from the malfunction of an RSU or a series of VANETs.

The IDS can act like the first-line of defence in case of an attack. However, the IDS might not be able to deal with more complex attacks such as ARP (Address Resolution Protocol) poisoning. More needs to be done in that respect for improving the proposed IDS. The final level in the IDS is any government agency [8] manning the entire network, however, no step has been proposed if this agency itself gets hacked. These agencies essentially monitor the entire network, and their losing control of the system can lead to widespread panic and catastrophe.

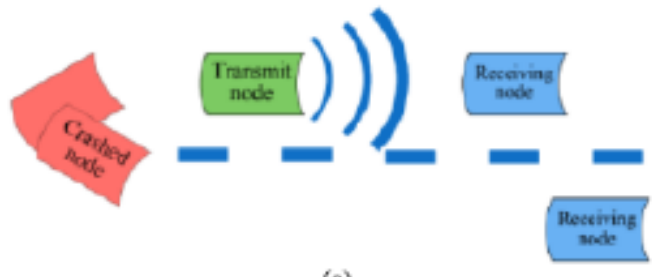

(a)

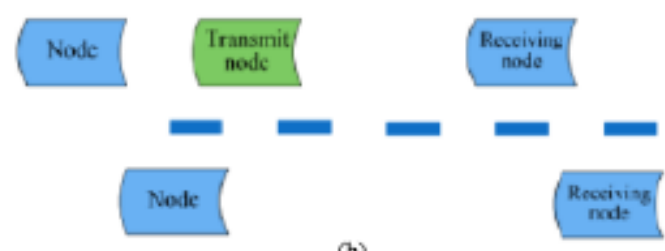

(a) Emergency Braking

(b) False Emergency Braking

Figure 4: Braking System [13] 


\section{CONCLUSION}

The concept of autonomous machines has successfully made it in the industry but the concept of an autonomous vehicle is still work in progress. Self-Driving vehicles are less of individualistic machines and more like nodes of a network. One of the major advantages of self-driving cars is that they can communicate and make decisions that are not just beneficial to them but beneficial to an entire group of vehicles in the vicinity. The goal of this paper was to bring light on the different aspects of a self-driving car. The ideas in handling of navigating an autonomous vehicle are new and varied. There is immense scope for improvement and innovation as well, as there is no set path on which methods of navigation can be based. Security-wise, the IDS will act like the first line of defence, but the end goal is catch-all the threats and neutralizes them before catastrophe strikes. Even after years of research into the different aspects, more needs to be done before a completely functional system of inter-connected selfdriving cars can be implemented safely. Self-driving vehicles not only bring about a change in the way we drive but they practically reanimate the entire transportation industry by bringing all the different kinds of transportation in sync and to strike a balance in them is a task that is yet to be completed by us. However, self-driving cars are poised to increase the efficiency and safety of the road in the not-so-distant future.

\section{REFERENCES}

[1] Shahroz Tariq, Hyunsoo Choi, C.M. Wasiq, Heenim Park "Controlled Parking for Self-Driving Cars" IEEE International Conference on Systems, Man and Cybernetics 2016, pp.1861-1865

[2] World Health Organization Global status report on Road Safety 2015

[3] Ungui Lee, Jiwon Jung, Seunghak Shin, Yongseop Jeong, Kibaek Park, David Hyunchul Shim, In-so Kweon "EureCar Turbo: a SelfDriving Car that can Handle Adverse Weather Conditions" IEEE/RSJ International Conference on Intelligent Robots and Systems (IROS) 2016, pp. 2301-2306

[4] T.Shim, G.Adireddy and H.Yuan "Autonomous vehicle collision avoidance system using path planning and model-predictive-control-based active front steering and wheel torque control" Journal of Automobile Engineering 2012, pp. 275-283

[5] Zhilu Chen and Xinming Huang "End-to-End Learning for Lane Keeping of Self-Driving Cars" IEEE Intelligent Vehicles Symposium (IV) 2017, pp.1856-1860
Jianwei Gong, Shengyue Yuan, Jiang Yan, Xuemei Chen, Huijun Di "Intuitive Decision-making Modelling for Self-driving Vehicles" IEEE 17th International Conference on Intelligent Transportation Systems (ITSC) 2014, pp.29-34

[6] Khattab M. Ali Alheeti, Anna Gruebler and Klaus D. McDonaldMaier "A model based path planning algorithm for self-driving cars in dynamic environment" Sixth International Conference on Emerging Security Technologies 2015, pp. 86-91

[7] Alberto Y. Hata, Fernando S. Osorio and Denis F. Wolf "Robust Curb Detection and Vehicle Localization in Urban Environments" 2014 IEEE Intelligent Vehicles Symposium (IV) pp. 1257-1262

[8] Unghui Lee, Sangyol Yoon, HyunChul Shim, Pascal Vasseur and Cedric Demonceaux "Local path planning in a complex environment for selfdriving car" The $4^{\text {th }}$ Annual IEEE International Conference on Cyber Technology in Automation, Control, and Intelligent Systems Pp. 445-450

[9] Pascale-L. Blyth, Milo N. Mladenovi, Bonnie A. Nardi, Norman M. Su, Hamid R. Ekbia "Driving the SelfDriving Vehicle" IEEE International Symposium on Technology in Society (ISTAS) Proceedings 2015 ISBN978-1-4799-8283-7

[10] Jeremy Straub, Wafaa Amer, Christian Ames, Karanam Ravichandran Dayananda, Andrew Jones, Goutham Miryala, Dylan Shipman "An Internetworked SelfDriving Car System-of-Systems" 2017 12th System of Systems Engineering Conference (SoSE) Pp.

[11] Patrick Y. Shinzato, Denis F. Wolf, and Christoph Stiller "Road Terrain Detection: Avoiding Common Obstacle Detection Assumptions Using Sensor Fusion" 2014 IEEE Intelligent Vehicles Symposium (IV) pp. 687-692

[12] Jeremy Straub, John McMillan, Brett Yaniero, Mitchell Schumacher, Abdullah Almosalami, Kelvin Boatey and Jordan Hartman "CyberSecurity considerations for an interconnected self-driving car system of systems" 2017 12th System of Systems Engineering Conference (SoSE)

[13] Anna Gruebler, Klaus D.McDonald-Maier, and Khattab M.Ali Alheeti "An Intrusion Detection System Against Black Hole Attacks on the Communication Network of Self-Driving Cars" 2015 Sixth International Conference on Emerging Security Technologies pp. 86-91 